\title{
Overexpression of cofilin 1 in prostate cancer and the corresponding clinical implications
}

\author{
$\mathrm{LI} \mathrm{LU}^{1 *}, \mathrm{NI} \mathrm{FU}^{2 *}, \mathrm{XU} \mathrm{LUO}^{2}, \mathrm{XIAO-YUN} \mathrm{LI}{ }^{1}$ and XIAO-PING LI ${ }^{1}$ \\ ${ }^{1}$ Department of Translational Medicine, Zhongshan School of Medicine, \\ Sun Yat-sen University, Guangzhou, Guangdong 510080; ${ }^{2}$ Department of Urology, \\ The Affiliated Hospital of Zunyi Medical College, Zunyi, Guizhou 560310, P.R. China
}

Received June 30, 2014; Accepted March 26, 2015

DOI: $10.3892 / \mathrm{ol} .2015 .3133$

\begin{abstract}
Cofilin 1 (CFL1) is a cytoskeletal protein and overexpression of the protein has been associated with aggressiveness in certain types of malignancies. The aim of the present study was to investigate the clinical implications of CFL1 expression in prostate cancer (PCa). Immunohistochemical analysis was performed using formalin-fixed paraffin-embedded tissue sections obtained from 111 patients with $\mathrm{PCa}$ and 47 patients with benign prostatic hyperplasia (BPH). In total, 78 (70.3\%) out of $111 \mathrm{PCa}$ tissues were found to express the CFL1 protein, while no expression was detected in BPH tissues. In addition, CFL1 was also observed to be significantly associated with the Gleason score (GS; $<7$ vs. $\geq 7$; $\mathrm{P}<0.0001$ ) and presence of lymph node metastasis (presence vs. absence; $\mathrm{P}<0.0001$ ). However, there was no association between the expression of CFL1 and other clinicopathological variables, such as age ( $<69$ years vs. $\geq 69$ years; $P=0.54$ ), pre-operative prostate specific antigen level $(<20 \mathrm{ng} / \mathrm{ml}$ vs. $\geq 20 \mathrm{ng} / \mathrm{ml} ; \mathrm{P}=0.45)$ and pathological stage (T2 vs. $\geq \mathrm{T} 3 \mathrm{a} ; \mathrm{P}=0.055)$. In addition, 35 tissues $(31.5 \%)$ were observed to possess a CFL1-positive mesenchyme. CFL1 expression was revealed to be an independent predictive factor for a high GS. The status of CFL1 expression in the mesenchyme also found to individually predict extraprostatic extension in PCa patients, based on multivariate analysis. The results of the present study indicated that CFL1 may specifically predict the development of PCa, and that the expression of CFL1 in the mesenchyme may be closely associated with the development of lymph node metastasis.
\end{abstract}

Correspondence to: Dr Xiao-Ping Li, Department of Translational Medicine, Zhongshan School of Medicine, Sun Yat-sen University, 74 Zhong Shan Road, Guangzhou, Guangdong 510080, P.R. China

E-mail: kinase001@aliyun.com

"Contributed equally

Key words: cofilin 1, immunohistochemistry, prostate cancer, prostatic hyperplasia, clinical implications

\section{Introduction}

Prostate cancer $(\mathrm{PCa})$ is one of the most common non-cutaneous malignancies in males. Since the introduction of the prostate specific antigen (PSA)-based screening strategy in clinical practice, a marked increase in the incidence of $\mathrm{PCa}$ has been observed (1). Although the use of this screening strategy has resulted in a $40 \%$ reduction in $\mathrm{PCa}$-associated mortality, the majority of patients succumb to the disease once metastasis has occurred. In addition, overtreatment of indolent PCa has emerged. This phenomenon may account for the deficiencies in accurate diagnosis and risk stratification. Therefore, the identification and validation of novel biomarkers for $\mathrm{PCa}$ should be considered a priority (2).

Cofilin 1 (CFL1) is the non-muscle isoform of the product of the CFL1 gene (Gene ID, 1072). CFL1 is a small ubiquitous protein that is able to bind monomeric globular $(\mathrm{G})$ and filamentous actin and inhibits the polymerization of monomeric $\mathrm{G}$-actin in a $\mathrm{pH}$-dependent manner (3), playing a key role in cell migration and cytokinesis (4). This protein is reported to be directly associated with the invasion, metastasis and chemoresistance of various human malignant solid tumors $(5,6)$. However, no previous studies regarding CFL1 expression and its association with clinicopathological features in PCa are available in the literature. The expression of CFL1 and its clinical implications in PCa are investigated in the present study.

\section{Materials and methods}

Patient characteristics and specimens. In total, 111 patients with histologically confirmed prostatic adenocarcinoma were enrolled in the present study. The patients had undergone open radical prostatectomy in the Department of Urology in The Affiliated Hospital of Zunyi Medical College (Zunyi, Guizhou) between January 2002 and September 2012. No patients received adjuvant androgen deprivation therapy prior to surgery. The histological analysis of all cancer specimens was conducted according to the Gleason score (GS) grading system (7) prior to immunohistochemical analysis. In addition to the PCa samples, 47 corresponding benign prostatic hyperplasia (BPH) tissues were selected as controls. The mean age of patients at the time of diagnosis was 
69 years (range, $51-81$ years). In total, 89 patients possessed no lymph node metastases and the mean pre-operative PSA level was measured as $19.97 \mathrm{ng} / \mathrm{ml}$ (range, 0.14-98 ng/ml). For the 47 patients diagnosed with $\mathrm{BPH}$, the mean age was 68 years (range, 52-79 years) and the mean PSA level was $11.0 \mathrm{ng} / \mathrm{ml}$ (range, $0.3-25.4 \mathrm{ng} / \mathrm{ml}$ ).

The use of the aforementioned tissues was approved by the Institutional Review Board of The Affiliated Hospital of Zunyi Medical College, and written informed consent was obtained from all patients.

Histological staining and immunohistochemical analysis. Paraffin-embedded 4-mm thick tissue sections were prepared from all samples for histological analysis. The tissue sections were stained with hematoxylin and eosin prior to the immunohistochemical detection of the CFL1 protein using a rabbit polyclonal anti-human CFL1 primary antibody (bs-2759R, dilution, 1:200; Bioss, Inc., Woburn, MA, USA).

All tissue sections were dewaxed, rehydrated and incubated in $3 \%$ hydrogen peroxide for $10 \mathrm{~min}$ at room temperature to quench endogenous peroxidase activity. The sections were then incubated overnight with the CFL1 antibody at $4^{\circ} \mathrm{C}$ in phosphate buffered saline (PBS) containing $1 \%$ bovine serum albumin. Staining was detected using an EnVision kit (ZSGB-Bio, Beijing, China) and 3,3'-diaminobenzidine (DAB; ZSGB-Bio) with $0.3 \% \mathrm{H}_{2} \mathrm{O}_{2}$ in PBS was used as the chromogen. Subsequent to staining, the sections were counterstained using hematoxylin and then dehydrated using ethanol and xylene, and Permount mounting medium was applied to the coverslips (all from Nanjing KeyGen Biotech. Co. Ltd., Shanghai, China). Rat immunoglobulin G primary antibody (CB3560554, dilution, 1:200; Biomeda Corporation, Foster City, CA, USA) was used as the negative control.

Imaging and statistical analysis. Histological analysis was redetermined simultaneously by two investigators using a double-headed light microscope. Evaluation of CFL1 expression was scored according to the percentage of positively stained cells in the field of view, as follows: Negative (0), no staining; weak (+), $0-33 \%$ of cells stained; moderate $(++)$, $34-66 \%$ of cells stained; and strong $(+++) 67-100 \%$ of cells stained. The association between CFL1 expression and clinicopathological parameters was analyzed by $\chi^{2}$ or Fisher's exact tests. Factors corresponding with the GS grouping or extraprostatic extension were analyzed using the logistic regression method. SPSS software version 13.0 (SPSS, Inc., Chicago, IL, USA) was used for the statistical analyses. $\mathrm{P}<0.05$ was considered to indicate a statistically significant difference.

\section{Results}

CFL1 expression in PCa and BPH samples. Expression of CFL1 was observed in $78(70.3 \%)$ PCa tumors, and no CFL1 overexpression was detected in BPH samples. Additionally, 35 lesions (31.6\%) exhibited light staining, 40 lesions (36.0\%) exhibited moderate staining and three lesions (2.7\%) exhibited strong staining for CFL1. Microscopically, it was observed that CFL1 was expressed in the cytoplasm, with low to high expression in PCa cancer cells. The expression of CFL1 was also observed in PCa cells located in the mesenchyme (Fig. 1B-E): CFL1 expression was increased in patients that underwent lymph node metastasis $(62.9 \%$; 22 out of 35 patients; Table I). The distribution of the CFL1 staining intensity and the expression of CFL1 in the mesenchyme of all PCa samples is shown in Fig. 2A and B.

Association between the expression of CFL1 and clinicopathologicalfeatures. Overexpression of CFL1 was revealed to differ significantly between patients with a post-operative GS $<7$ and patients with a $\mathrm{GS} \geq 7$ (50 vs. $86.9 \%$, respectively; $\mathrm{P}<0.0001$ ). A similar incidence of overexpression was observed in patients with lymph node metastasis compared with those without (100 vs. $62.9 \%$, respectively; $\mathrm{P}<0.0001)$. The CFL1 expression rate in patients $\geq 69$ years of age was not significantly different from that of patients aged $<69$ years $(67.3$ vs. $72.9 \%$, respectively; $\mathrm{P}=0.54)$. Overexpression of $\mathrm{CFL} 1$ also did not differ between patients with a PSA level of $<20 \mathrm{ng} / \mathrm{ml}$ and those with a level of $\geq 20 \mathrm{ng} / \mathrm{ml}$ (68 vs. $75 \%$, respectively; $\mathrm{P}=0.45$ ), or between patients with stage pT2 tumors and those with pT3-4 (extraprostatic extension) tumors (63.2 vs. $81.4 \%$, respectively; $\mathrm{P}=0.055$ ) (Table $\mathrm{I}$ ).

Binary logistic analysis was further conducted for the predictive ability of all factors in order to analyze whether CFL1 expression was significantly associated with clinicopathological features (Table II). PSA level [relative risk, 1.076; 95\% confidence interval (CI), 1.034-1.121; $\mathrm{P}<0.0001]$, CFL1 overexpression (relative risk, 6.625; 95\% CI, 2.621-16.747; $\mathrm{P}<0.0001$ ) and CFL1-positive mesenchyme cells (relative risk, 6.646; 95\% CI, 2.469-17.885; $\mathrm{P}<0.0001$ ) were all significantly associated with a high GS at the univariate level. Similarly, a strong association was observed between extraprostatic extension ( $\geq$ pT3a) and PSA level (relative risk, 1.095; 95\% CI, 0.935-1.053; $\mathrm{P}<0.0001$ ), post-operative GS (relative risk, 2.731; 95\% CI, 1.917-3.890; $\mathrm{P}<0.0001)$, CFL1 expression (relative risk, 2.820; 95\% CI, 1.133-7.019; $\mathrm{P}=0.026$ ) and CFL1-positive mesenchyme cells (relative risk, 10.875; 95\% CI, 4.207-28.114; $\mathrm{P}<0.0001$ ).

Multivariate analysis revealed that the PSA level (relative risk, 1.087; 95\% CI, 1.034-1.144; $\mathrm{P}<0.0001)$ and CFL1 expression (relative risk, 5.287; 95\% CI, 1.627-17.177; $\mathrm{P}=0.006$ ) were independent predictors of high GS, regardless of age and mesenchymal CFL1 expression. In addition, PSA (relative risk, 1.070; 95\% CI, 1.024-1.118; $\mathrm{P}=0.002$ ), post-operative GS (relative risk, 2.280; 95\% CI, 1.516-3.430; $\mathrm{P}<0.0001$ ) and mesenchymal CFL1 status (relative risk, 9.143; 95\% CI, 2.187-38.228; $\mathrm{P}=0.002$ ) were found to be independent factors predictive of extraprostatic extension ( $\geq \mathrm{T} 3 \mathrm{a}$ stage) at the multivariate level, while age was not a significant predictor of extraprostatic extension.

\section{Discussion}

The exploration of novel biomarkers is of practical significance for PCa, as it may inform physicians and surgeons of which patients require radical surgery or active surveillance. It may also facilitate improved screening, diagnosis, clinical outcome prediction and decision making prior to surgery (8). Due to the high incidence of PCa worldwide, there is an urgent demand for the identification of robust biomarkers. Although PSA remains as the most widely used biomarker for the diagnosis 
Table I. Clinicopathological parameters of patients from whom samples were obtained.

\begin{tabular}{|c|c|c|c|}
\hline Variables & PCa, n & CFL1 positive in $\mathrm{PCa}, \mathrm{n}(\%)$ & P-value \\
\hline Age, years & & & 0.54 \\
\hline$<69$ & 59 & $43(72.9)$ & \\
\hline$\geq 69$ & 52 & $35(67.3)$ & \\
\hline Preoperative PSA, ng/ml & & & 0.45 \\
\hline$<20$ & 75 & $51(68.0)$ & \\
\hline$\geq 20$ & 36 & $27(75.0)$ & \\
\hline Pathological stage & & & 0.055 \\
\hline $\mathrm{T} 2 \mathrm{a} / 2 \mathrm{~b} / 2 \mathrm{c}$ & 68 & $43(63.2)$ & \\
\hline$\geq \mathrm{T} 3 \mathrm{a}$ & 43 & $35(81.4)$ & \\
\hline Postoperative GS & & & $<0.0001$ \\
\hline$<7$ & 50 & $25(50.0)$ & \\
\hline$\geq 7$ & 61 & $53(86.9)$ & \\
\hline Lymph node metastasis & & & $<0.0001$ \\
\hline No & 89 & $56(62.9)$ & \\
\hline Yes & 22 & $22(100.0)$ & \\
\hline
\end{tabular}

PCa, prostate cancer; CFL1, cofilin 1; PSA, prostate specific antigen; GS, Gleason score.

Table II. Variables associated with GS and pathological stage stratification for prostate cancer.

\begin{tabular}{|c|c|c|c|c|c|c|}
\hline \multirow[b]{2}{*}{ Variables } & \multicolumn{3}{|c|}{ GS, $<7$ vs. $\geq 7$} & \multicolumn{3}{|c|}{ Pathological stage, T2 vs. $\geq \mathrm{T} 3 \mathrm{a}$} \\
\hline & P-value & RR & $95 \% \mathrm{CI}$ & P-value & RR & $95 \% \mathrm{CI}$ \\
\hline \multicolumn{7}{|l|}{ Univariate analysis } \\
\hline Age, $<69$ vs. $\geq 69$ years & 0.240 & 0.965 & $0.908-1.024$ & 0.800 & 0.992 & $0.935-1.053$ \\
\hline PSA, $<20 \mathrm{vs} . \geq 20 \mathrm{ng} / \mathrm{ml}$ & $<0.0001$ & 1.076 & $1.034-1.121$ & $<0.0001$ & 1.095 & $0.935-1.053$ \\
\hline Post-op GS, $<7$ vs. $\geq 7$ & - & - & - & $<0.0001$ & 2.731 & $1.917-3.890$ \\
\hline Cofilin 1, pos vs. neg & $<0.0001$ & 6.625 & $2.621-16.747$ & 0.026 & 2.820 & $1.133-7.019$ \\
\hline Mesen status, pos vs. neg & $<0.0001$ & 6.646 & $2.469-17.885$ & $<0.0001$ & 10.875 & $4.207-28.114$ \\
\hline \multicolumn{7}{|l|}{ Multivariate analysis } \\
\hline Age, $<69$ vs. $\geq 69$ years & 0.334 & 0.962 & $0.890-1.041$ & 0.510 & 1.033 & $0.938-1.136$ \\
\hline PSA, $<20 \mathrm{vs} . \geq 20 \mathrm{ng} / \mathrm{ml}$ & 0.001 & 1.087 & $1.034-1.144$ & 0.002 & 1.070 & $1.024-1.118$ \\
\hline Post-op GS, $<7$ vs. $\geq 7$ & - & - & - & $<0.0001$ & 2.280 & $1.516-3.430$ \\
\hline Cofilin 1, pos vs. neg & 0.006 & 5.287 & $1.627-17.177$ & 0.184 & 0.347 & $0.073-1.654$ \\
\hline Mesen status, pos vs. neg & 0.105 & 2.619 & $0.817-8.399$ & 0.002 & 9.143 & $2.187-38.228$ \\
\hline
\end{tabular}

RR, relative risk; CI, confidence interval; PSA, prostate specific antigen; post-op, postoperative; GS, Gleason score; pos, positive; neg, negative; mesen, mesenchyme.

and screening of $\mathrm{PCa}$, it demonstrates a number of limitations, including the occurrence of false-positive diagnosis and over-treatment due to the poor sensitivity and specificity of PSA level testing (9-11).

In the present study, $70.3 \%$ of PCa cases were found to be positive for CFL1 expression, with expression predominantly observed in the cytoplasm of cancer cells. CFL1-positive cancer cells were also observed in the mesenchyme in all cases with lymph node metastasis. The rate of positive CFL1 expression was increased significantly in poorly-differentiated PCa, defined by a $\mathrm{GS} \geq 7$ or the presence of lymph node metastasis. Furthermore, CFL1 expression was absent in BPH tissues. Therefore, CFL1 immunohistochemical expression is specific to $\mathrm{PCa}$, and is associated with the aggressiveness of the phenotype. This is consistent with a number of studies that have reported CFL1 to be associated with a more aggressive phenotype and with tumor progression in various solid tumor tissues (12-15). For instance, CFL1 has been reported to play a major role in tumor progression in ovarian carcinomas, as nearly $64 \%$ of all ovarian tumors are positive for CFL1 (16), 


\section{A}
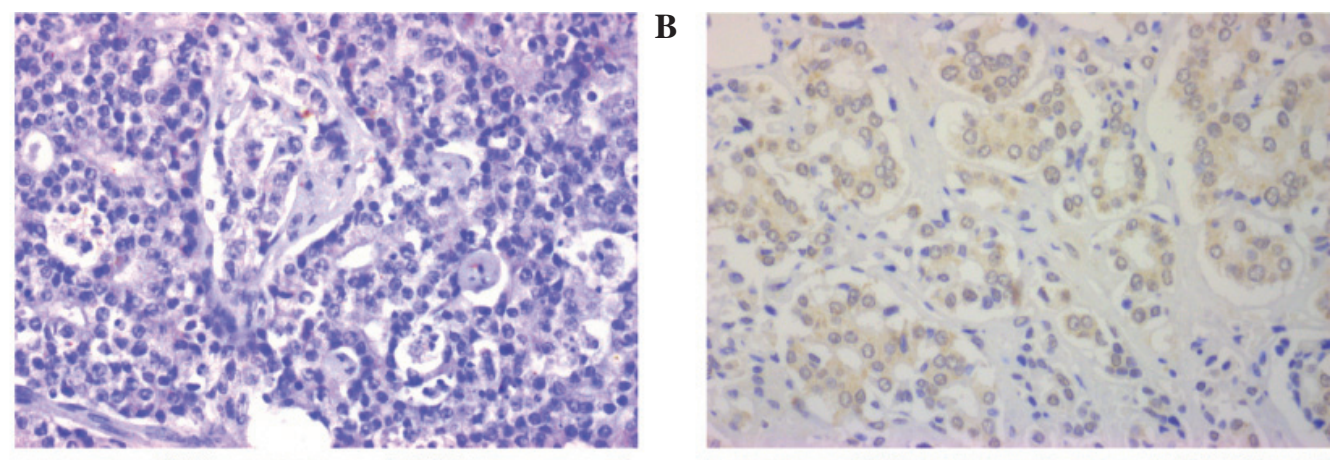

C
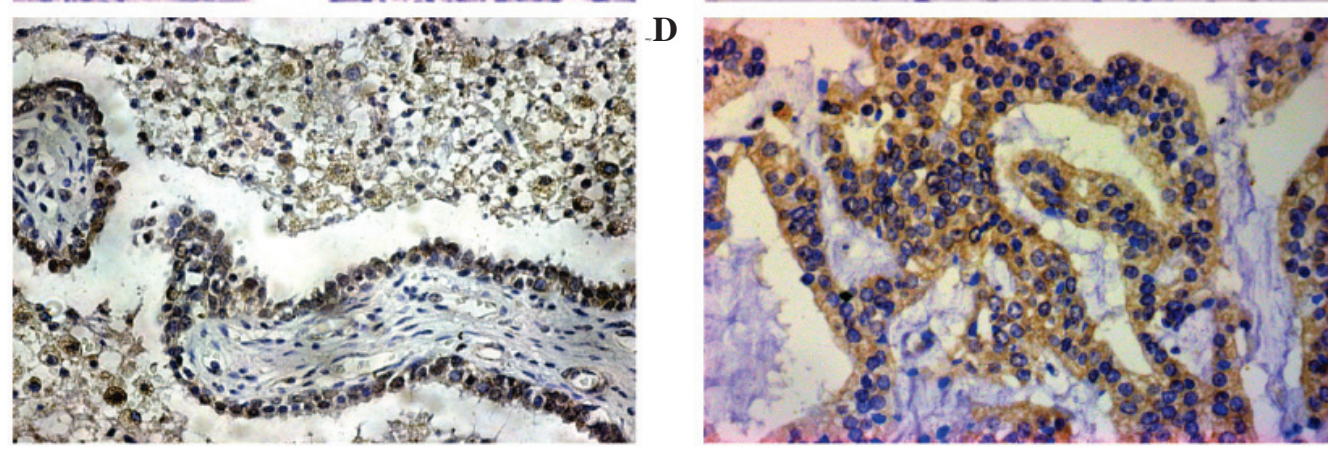

$\mathbf{E}$

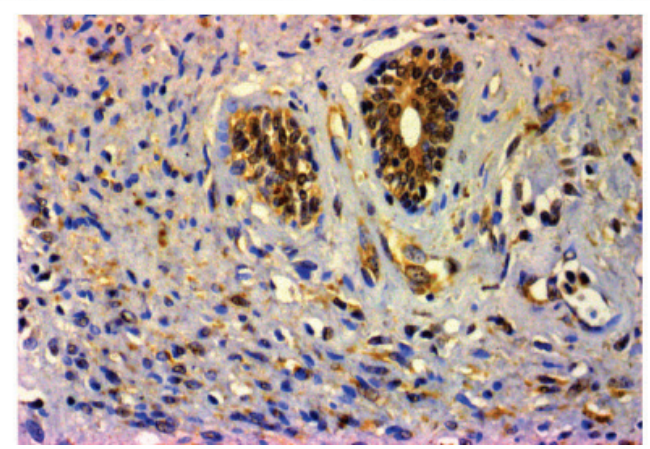

Figure 1. Prostate cancer specimens stained immunohistochemically for CFL1. (A) CFL1-negative cancer cells in prostate cancer specimen. Representative images of (B) weak, (C) moderate and (D) strong immunohistochemical expression of CFL1 in prostate cancer tissue specimens. (E) The immunohistochemical expression of CFL1 in the mesenchyme of a prostate cancer tissue specimen. Magnification, x400. CFL1, cofilin 1.
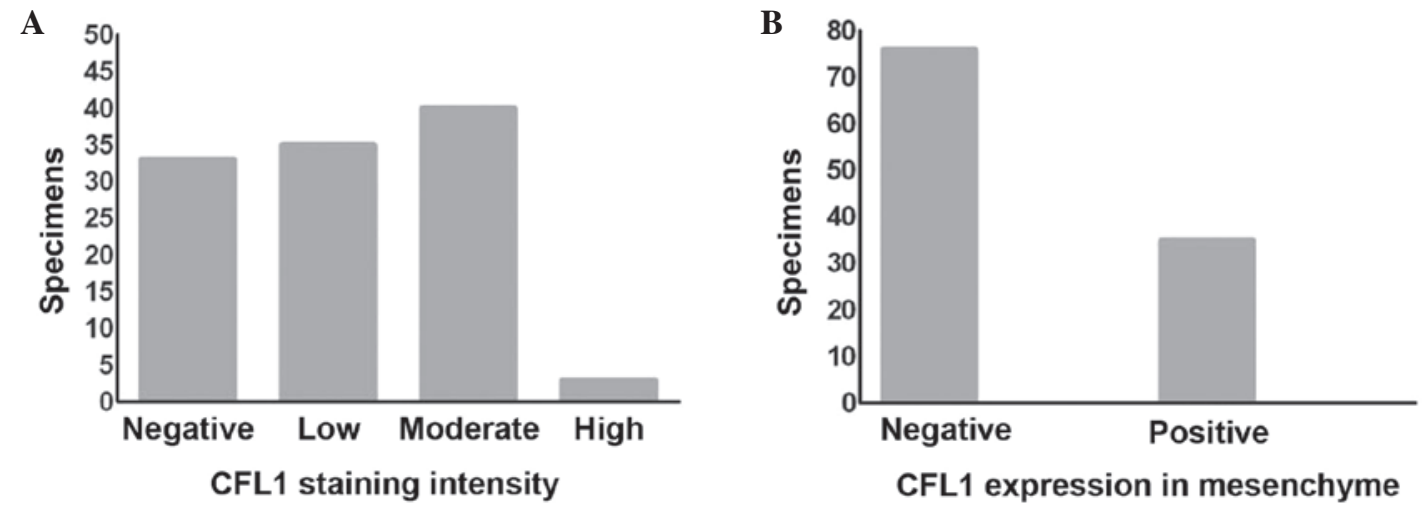

Figure 2. (A) Intensity of staining for CFL1 in prostate cancer tissues. (B) Staining for CFL1 in the mesenchyme of patients with prostate cancer. CFL1, cofilin 1.

upregulation of phosphorylated CFL1 levels result in increased chemoresistance (17) and the patients with CFL1-positive tumors demonstrate decreased progression-free survival rates compared with the patients with CFL1-negative lesions (18). A similar association is observed in urological carcinoma. Chung et al (19) reported that the invasiveness of bladder carcinomas is markedly enhanced in vitro subsequent to
CFL1 phosphorylation by endothelial growth factor. Furthermore, in PCa, CFL1 may also inhibit cancer cell growth by inducing the formation of cofilin-actin rods within the cancer cells (20), and knockdown of CFL1 results in the increased sensitivity of PCa to certain chemotherapeutic agents, including docetaxel (21). CFL1 is also important in the regulation of cancer cell migration and invasion capability $(6,22,23)$. 
Hotulainen et al (4) reported that the inhibition of cofilin activity was able to inhibit cell motility, while the overexpression of cofilin increased the velocity of cell migration in human glioblastoma cells (24).

Based on the logistic analysis, PSA and CFL1 were identified as the most important predictive factors for patients with a $\mathrm{GS} \geq 7$ subsequent to surgery. Similarly, the findings indicated that extra-prostatic extension in $\mathrm{PCa}$ was predicted by PSA levels, post-operative GS, CFL1 expression and CFL1 status in the mesenchyme. In general, PSA is positively associated with a higher GS and continues to be a strong predictor of extraprostatic extension $(25,26)$. However, factors such as race or ethnicity may significantly affect PSA values, even after adjustment for age and prostate volume (27-29). A number of studies have also demonstrated that a high GS is useful for predicting extraprostatic extension (30-32), and the present findings were consistent with these findings. As CFL1 expression was an independent prognostic factor in $\mathrm{PCa}$, immunohistochemical detection of this marker in cancer tissue samples may aid in decision making. However, the exact mechanism of CFL1 in tumor pathogenesis and invasion requires additional investigation.

In conclusion, the present findings of the evaluation of CFL1 as a biomarker revealed that this molecule has high specificity in distinguishing malignant prostate tissues from $\mathrm{BPH}$, which may help to avoid the misdiagnosis of $\mathrm{BPH}$ as PCa. CFL1 expression was also found to be strongly associated with aggressive characteristics, and may occur even before cancer cell initiation and invasion. Although further studies are necessary, CFL1 is a promising target that may be used as biomarker for early diagnosis, monitoring, and decision making for treatment.

\section{Acknowledgements}

The authors would like to thank the Department of Pathology at The Affiliated Hospital of Zunyi Medical College.

\section{References}

1. Siegel R, Naishadham D and Jemal A: Cancer statistics, 2013 CA Cancer J Clin 63: 11-30, 2013.

2. Kelloff GJ and Sigman CC: Cancer biomarkers: selecting the right drug for the right patient. Nat Rev Drug Discov 11: 201-214, 2012.

3. Ono S: Mechanism of depolymerization and severing of actin filaments and its significance in cytoskeletal dynamics. Int Rev Cytol 258: 1-82, 2007.

4. Hotulainen P, Paunola E, Vartiainen MK and Lappalainen P: Actin-depolymerizing factor and cofilin-1 play overlapping roles in promoting rapid F-actin depolymerization in mammalian nonmuscle cells. Mol Biol Cell 16: 649-664, 2005.

5. Zhu B, Fukada K, Zhu H and Kyprianou N: Prohibitin and cofilin are intracellular effectors of transforming growth factor beta signaling in human prostate cancer cells. Cancer Res 66: 8640-8647, 2006.

6. Wang W, Mouneimne G, Sidani M, et al: The activity status of cofilin is directly related to invasion, intravasation and metastasis of mammary tumors. J Cell Biol 173: 395-404, 2006.

7. Epstein JI: An update of the Gleason grading system. J Urol 183 433-440, 2010.

8. Prensner JR, Rubin MA, Wei JT and Chinnaiyan AM: Beyond PSA: the next generation of prostate cancer biomarkers. Sci Transl Med 4: 127rv3, 2012.

9. Lilja H, Ulmert D and Vickers AJ: Prostate-specific antigen and prostate cancer: prediction, detection and monitoring. Nat Rev Cancer 8: 268-278, 2008.
10. Wolf AM, Wender RC, Etzioni RB, et al; American Cancer Society Prostate Cancer Advisory Committee: American Cancer Society guideline for the early detection of prostate cancer: update 2010 . CA Cancer J Clin 60: 70-98, 2010.

11. Balk SP, Ko YJ and Bubley GJ: Biology of prostate-specific antigen. J Clin Oncol 21: 383-391, 2003.

12. Castro MA, Dal-Pizzol F, Zdanov S, et al: CFL1 expression levels as a prognostic and drug resistance marker in nonsmall cell lung cancer. Cancer 116: 3645-3655, 2010.

13. Wang LH, Xiang J, Yan M, et al: The mitotic kinase Aurora-A induces mammary cell migration and breast cancer metastasis by activating the Cofilin-F-actin pathway. Cancer Res 70: 9118-9128, 2010.

14. Polachini GM, Sobral LM, Mercante AM, et al: Proteomic approaches identify members of cofilin pathway involved in oral tumorigenesis. PLoS One 7: e50517, 2012.

15. Yang ZL, Miao X, Xiong L, et al: CFL1 and Arp3 are biomarkers for metastasis and poor prognosis of squamous cell/adenosquamous carcinomas and adenocarcinomas of gallbladder. Cancer Invest 31: 132-139, 2013.

16. Zhou J, Wang Y, Fei J and Zhang W: Expression of cofilin 1 is positively correlated with the differentiation of human epithelial ovarian cancer. Oncol Lett 4: 1187-1190, 2012.

17. Nishimura S, Tsuda H, Kataoka F, et al: Overexpression of cofilin 1 can predict progression-free survival in patients with epithelial ovarian cancer receiving standard therapy. Hum Pathol 42: 516-521, 2011.

18. Li M, Yin J, Mao N and Pan L: Upregulation of phosphorylated cofilin 1 correlates with taxol resistance in human ovarian cancer in vitro and in vivo. Oncol Rep 29: 58-66, 2013.

19. Chung H, Kim B, Jung SH, et al: Does phosphorylation of cofilin affect the progression of human bladder cancer? BMC Cancer 13: 45,2013

20. Ren S, Ouyang DY, Saltis M, et al: Anti-proliferative effect of 23,24-dihydrocucurbitacin F on human prostate cancer cells through induction of actin aggregation and cofilin-actin rod formation. Cancer Chemother Pharmacol 70: 415-424, 2012.

21. Pérez-Martínez FC, Carrión B, Lucío MI, et al: Enhanced docetaxel-mediated cytotoxicity in human prostate cancer cells through knockdown of cofilin-1 by carbon nanohorn delivered siRNA. Biomaterials 33: 8152-8159, 2012.

22. van Rheenen J, Song X, van Roosmalen W, et al: EGF-induced PIP2 hydrolysis releases and activates cofilin locally in carcinoma cells. J Cell Biol 179: 1247-1259, 2007.

23. Oser M and Condeelis J: The cofilin activity cycle in lamellipodia and invadopodia. J Cell Biochem 108: 1252-1262, 2009.

24. Yap CT, Simpson TI, Pratt T, Price DJ and Maciver SK: The motility of glioblastoma tumour cells is modulated by intracellular cofilin expression in a concentration-dependent manner. Cell Motil Cytoskeleton 60: 153-165, 2005.

25. Partin AW, Carter HB, Chan DW, et al: Prostate specific antigen in the staging of localized prostate cancer: influence of tumor differentiation, tumor volume and benign hyperplasia. J Urol 143: 747-752, 1990.

26. Paquette EL, Connelly RR, Sun L, Paquette LR and Moul JW: Predictors of extracapsular extension and positive margins in African American and white men. Urol Oncol 21: 33-38, 2003.

27. Asbell SO and Vijayakumar S: Racial differences in prostate-specific antigen levels in patients with local-regional prostate cancer. Prostate 31: 42-46, 1997.

28. Moul JW, Connelly RR, Mooneyhan RM, et al: Racial differences in tumor volume and prostate specific antigen among radical prostatectomy patients. J Urol 162: 394-397, 1999.

29. Resnick MJ, Canter DJ, Guzzo TJ, et al: Does race affect postoperative outcomes in patients with low-risk prostate cancer who undergo radical prostatectomy? Urology 73: 620-623, 2009.

30. Magheli A, Rais-Bahrami S, Trock BJ, et al: Prostate specific antigen versus prostate specific antigen density as a prognosticator of pathological characteristics and biochemical recurrence following radical prostatectomy. J Urol 179: 1780-1784, 2008.

31. Giannarini G, Scott CA, Moro U, Pertoldi B, Beltrami CA and Selli C: Are PSA density and PSA density of the transition zone more accurate than PSA in predicting the pathological stage of clinically localized prostate cancer? Urol Oncol 26: 353-360, 2008.

32. Nishimoto K, Nakashima J, Hashiguchi A, et al: Prediction of extraprostatic extension by prostate specific antigen velocity, endorectal MRI, and biopsy Gleason score in clinically localized prostate cancer. Int J Urol 15: 520-523, 2008. 